\title{
Autoconceito e Clima Criativo em Sala de Aula na percepção de alunos do ensino fundamental
}

\author{
DenisedeSaza Flèth- UnivesidadedeBrasilia, BrasiliaDF, Brasil \\ EuniceMaria Lima SorianodeAlencar - UniversidadedeBraślia, Braślia-DF, Brasil
}

\begin{abstract}
Resumo
O objetivo deste estudo foi examinar a percepção do clima de sala de aula para criatividade e 0 autoconceito de 504 alunos de $6^{\circ}$ ano do ensino fundamental. Os participantes responderam à Escala sobre Clima para a Criatividade em Sala de Aula e ao Perfil de Autopercepção para Crianças. Os resultados indicaram que os alunos apresentaram percepção do clima de sala de aula e autoconceito positivos. Foram observadas diferenças em relação a essas variáveis considerando-se tipo de escola e gênero. Os alunos de escolas particulares avaliaram de forma mais favorável o clima de sala de aula para criatividade do que os de escola pública. Alunos de escolas particulares, do gênero masculino, apresentaram um autoconceito mais positivo. 0 clima de sala de aula para criatividade na disciplina de língua portuguesa foi mais bem avaliado pelos alunos quando contrastado com o de matemática.
\end{abstract}

Palavasdhave Criatividade; Clima de sala de aula; Autoconceito; Ensino fundamental.

\section{Self-concept and Creative Environment in the Classroom according to Elementary School Students}

\begin{abstract}
The purpose of this study was to examine the perception of the classroom environment for creativity and self-perception of $5045^{\text {th }}$ grade students. The participants answered the Classroom Envronment for Creativity Scale and the SelfPerception Profile for Children. The results indicated that students had a positive classroom climate perception and selfperception. Differences were observed for these variables regarding the school type and gender. Private school students evaluated the classroom environment for creativity in a more favorable way than public school students. Male students from private school had a more positive self- perception. The classroom environment for creativity in the subjects of Portuguese language was better evaluated by students when contrasted with the environment of Mathematics.

Kegnards Creativity; Classroom environment; Self-perception; Elementary school.
\end{abstract}

\section{Autoconcepto y Clima Creativo en el aula en la percepción de alumnos de la enseñanza básica}

\begin{abstract}
Resumen
El objetivo de este estudio fue examinar la percepción del clima en el aula para creatividad y el autoconcepto de 504 alumnos de 6o año de la enseñanza básica. Los participantes respondieron a la Escala sobre Clima para la Creatividad en Aula y al Perfil de Autopercepción para Niños. Los resultados indicaron que los alumnos presentaron percepción del clima en el aula y autoconcepto positivos. Fueron observadas diferencias en relación a esas variables considerándose tipo de escuela y género. Los alumnos de escuelas privadas evaluaron de forma más favorable el clima en el aula para creatividad que los de escuela pública. Alumnos de escuelas privadas, del género masculino, presentaron un autoconcepto más positivo. El clima en el aula para creatividad en la disciplina de Lengua Portuguesa fue mejor evaluado por los alumnos cuando contrastado con el de Matemática.

Palabrasdave Creatividad; Clima en el aula; Autoconcepto; Enseñanza Básica.
\end{abstract}

Criatividade passou a ser considerada um fenômeno individual e social desde as últimas décadas do século passado. A partir de então, para se compreender como, por que e quando novas ideias são produzidas, tornou-se necessário examinar variáveis intrapessoais e ambientais. Tanto valores e crenças pessoais, como o contexto social, histórico e cultural, influenciam no tipo de comportamento ou produto reconhecido como criativo em uma dada sociedade (Wechsler, 2006). Csikszentmihalyi (1996, 1999) defende que 0 foco dos estudos em criatividade deve ser nos sistemas sociais e não apenas no indivíduo. Para esse autor, criatividade é um processo que resulta da interação de três fatores: indivíduo, domínio e campo. 0 primeiro fator envolve bagagem genética, características e experiências pessoais. O domínio consiste em um conjunto de regras e procedimentos de uma área do conhecimento, transmitido e compartilhado pela sociedade. Por campo, entende-se especialistas de uma área do conhecimento que decidem se uma ideia nova é ou não criativa. 0 indivíduo que tem oportunidades de conhecer a fundo um domínio terá mais chances de identificar consistências e propor novas abordagens, que serão incorporadas ou não à área de conhecimento, conforme avaliação dos especialistas. 0 ambiente no qual o indivíduo está inserido desempenha, portanto, um papel importante, porque pode estimular ou inibir o desenvolvimento do potencial criativo.

Um dos contextos sociais mais investigados no que diz respeito à promoção da criatividade é a escola (Amabile, 1996; Beghetto, 2009; Fleith \& Alencar, 2006; Wechsler \& Souza, 2011). Os resultados ressaltam a importância de um clima de sala de aula favorecedor da aprendizagem e das habilidades criativas dos alunos. Um clima criativo em sala de aula pode ser caracterizado por uma atmosfera de aceitação e respeito entre colegas e professores; oportunidades de reflexão sobre o assunto estudado, de elaboração de novas interpretações e de avaliação crítica do conteúdo 
ministrado; valorizacäo dos interesses e habilidades dos discentes; promoção da autonomia dos alunos; diversidade de estratégias pedagógicas; e apoio à expressão de ideias originais (Alencar \& Fleith, 2009; Cropley, 2005).

Evidências empíricas acerca da extensão em que 0 ambiente escolar favorece a criatividade $\mathrm{e}$ aprendizagem, na percepção de alunos, não são conclusivas. Por um lado, há estudos que sugerem que nem sempre o clima de sala de aula preferido pelos alunos coincide com o que eles vivenciam (Alencar \& Fleith, 2004; Fraser, 1984; Johnson \& Johnson, 1999). Por outro, pesquisas têm sinalizado uma avaliação positiva de alunos do ensino fundamental sobre 0 clima para criatividade em sala de aula, especialmente aqueles motivados pelas atividades escolares (PinheiroCavalcanti, 2009), os provenientes de escolas particulares (Fleith \& Alencar, 2006) e aqueles cujas professoras têm mais tempo de experiência docente (Castro \& Fleith, 2008).

Com relação aos fatores individuais que contribuem para a expressão criativa, Selby, Shaw e Houtz (2005) apontam várias características, tais como sensibilidade a problemas, curiosidade, abertura a emoções e sentimentos, independência de pensamento, autonomia, persistência e autoconceito positivo. Esquivel e Hodes (2003) chamam a atenção para a correlação positiva entre criatividade e maturidade social, assertividade e autoimagem positiva. O autoconceito pode ser considerado uma variável que agrega várias dimensões e que facilita a emergência de outras variáveis, como a criatividade. Para Byrne (1996), "autoconceito é conceituado ao longo de um continuumde percepções muito específicas a globais do indivíduo acerca de suas competências, e essas abordagens não são mutuamente exclusivas" (p. 16). Embora a relação entre criatividade e autoconceito seja defendida por muitos autores, os estudos empíricos a respeito dessa associação são limitados e os resultados obtidos são divergentes, conforme relatam Fleith, Renzulli e Westberg (2002) e Hennessay e Amabile (1988).

O papel ativo do indivíduo no processo criativo, bem como a influência dos fatores sociais, culturais e históricos na produção criativa e na avaliação do trabalho criativo, é inquestionável. 0 ambiente escolar, em especial, tem sido destacado por inúmeros pesquisadores da área como aquele que mais impacto tem produzido na expressão criativa. Torrance (citado por Esquivel \& Hodes, 2003), por exemplo, chama a atenção para um declínio nos níveis de criatividade, particularmente no que diz respeito à originalidade de ideias, a par de um aumento no traço relacionado à conformidade de pensamento, em alunos de $5^{\circ}$ ano ${ }^{1}$ do ensino fundamental. Da mesma forma, Smith e Carlsson (citados por Cropley, 2005) apontam uma descontinuidade no desenvolvimento da criatividade entre 10 e 16 anos de idade.

A partir desse cenário, desenvolveu-se este estudo com o objetivo de examinar a percepção do clima de sala de aula para criatividade e 0 autoconceito de alunos, do sexo masculino e feminino, de $6^{\circ}$ ano do ensino fundamental, de escolas públicas e particulares. Tendo em vista que os alunos cursam várias disciplinas ministradas por diferentes professores, decidiu-se concentrar nas de Língua Portuguesa e Matemática, por constituírem disciplinas centrais na formação do aluno. A pesquisa buscou ainda investigar a relação entre percepção do clima de sala de aula e autoconceito.

\section{Participantes}

\section{Método}

Participaram deste estudo 504 alunos de $6^{\circ}$ ano do ensino fundamental de cinco escolas públicas e quatro escolas particulares do Distrito Federal, sendo $224(44,4 \%)$ do gênero masculino e $279(55,4 \%)$ do feminino. Um aluno não respondeu a essa questão. Duzentos e noventa e oito alunos (59,1\%) frequentavam a escola pública, ao passo que 206 (40,9\%) eram provenientes de instituições particulares de ensino. A média de idade dos participantes era de 11,45 anos. Utilizou-se uma amostra de conveniência, dada a dificuldade de seleção randômica da amostra, uma vez que a participação dos sujeitos no projeto foi voluntária (Gall, Borg \& Gall, 1996).

\section{Instrumentos}

Clima para ciatividade em sala de aula A Escala sobre Clima para a Criatividade em Sala de Aula (Fleith \& Alencar, 2005), utilizada em estudos anteriores para identificar fatores associados à criatividade em sala de aula, segundo a percepção de alunos de $4^{\circ}$ e $5^{\circ}$ ano do ensino fundamental, foi adaptada e validada junto aos alunos de $6^{\circ}$ ano, participantes deste estudo (Fleith, Almeida \& Peixoto, 2011). Uma escala de frequência de 5 pontos é utilizada para a resposta aos itens: (1) nunca, (2) poucas vezes, (3) algumas vezes, (4) muitas vezes e (5) sempre. No presente estudo, essa escala foi respondida duas vezes pelos alunos - uma para avaliar o clima de sala de aula para criatividade em Língua Portuguesa e outra em Matemática.

\footnotetext{
1 A nova nomenclatura adotada pelo Ministério da Educação substitui série por ano. $05^{\circ}$ ano do ensino fundamental corresponde à antiga $4^{\mathrm{a}}$ série e $06^{0}$ ano à $5^{\mathrm{a}}$ série.
} 
Realizou-se uma análise fatorial confirmatória (Fleith \& cols., 2011). Ante o modelo testado, três dimensões associadas ao clima de sala de aula para criatividade, com 21 itens, considerando tanto a disciplina de Língua Portuguesa quanto de Matemática, foram confirmadas: Postura do Professor em Sala de Aula (8 itens), Interesse do Aluno pela Aprendizagem (7 itens) e Autopercepção do Aluno com Relação à Criatividade (6 itens). Postura do Professor em Sala de Aula refere-se à postura de incentivo e aceitação por parte do professor às ideias e produções dos alunos. Interesse do Aluno pela Aprendizagem trata do envolvimento do aluno com o trabalho escolar. Autopercepção do Aluno com Relação à Criatividade diz respeito à imagem que o aluno tem de si mesmo em relação ao seu nível de criatividade. Os índices obtidos, considerando os 21 itens da escala retidos, sugerem uma boa adequação do modelo na base dos índices estatísticos obtidos tanto no caso da disciplina Língua Portuguesa [CMIN/ DF $=2,54 ; \mathrm{GFI}=0,92 ; \mathrm{AGFI}=0,90$; TLI=0,91; RMSEA $=0,06$ ] quanto na de Matemática [CMIN/ D F=2,33; GFI=0,92; AGFI=0,90; TLI=0,93; RMSEA $=0,05]$. O s índices alfa de consistência interna variaram de 0,69 a 0,82 para Língua Portuguesa e de 0,72 a 0,88 para Matemática. Exemplos de itens da escala são: na minha sala de aula, o professor ouve as minhas ideias, eu tenho oportunidade de participar de várias atividades, eu uso minha imaginação, eu tenho ideias diferentes, eu aprendo coisas que realmente me interessam.

Autoconcita A escala Perfil de Autopercepção para Crianças (Harter, 1990) foi desenvolvida para avaliar 0 julgamento de crianças, de $4^{\circ}$ a 90 ano do ensino fundamental, sobre sua competência em domínios específicos e de maneira global: competência escolar, aceitação social, competência atlética, aparência física, conduta comportamental e autoestima global.

Cada subescala contém seis itens. Cada item inclui duas sentenças opostas descrevendo características de uma criança. A criança é instruída a decidir qual o tipo de criança é mais parecida com ela e, em seguida, é solicitada a indicar se sua resposta se aplica mais ou menos ou totalmente a ela. Cada item é avaliado em uma escala de 1 a 4 , onde 0 escore 1 indica percepção negativa de sua competência, enquanto o escore 4 indica uma percepção positiva de sua competência. Cada escala apresenta um escore final, obtido por meio da soma de pontos dos itens que a compõem (Harter, 1985). 0 número mínimo de pontos é 6 e 0 máximo é 24, por subescala. Esse instrumento foi selecionado para 0 estudo tendo em vista ser apropriado para a idade dos alunos participantes do estudo e citado frequentemente na literatura da área, inclusive no Brasil (Bandeira, Arteche \& Reppold, 2008; Ourofino \&
Fleith, 2005; Silva, 2002). Os índices alfa de consistência interna variam de 0,75 a 0,82 (Harter, 1985). Em um estudo de validação do instrumento para o Brasil, Valentini, Villwock, Vieira, Vieira e Barbosa (2010) encontraram bons índices de consistência interna teste-reteste, especialmente em amostra de alunos entre 11 e 12 anos de idade. Exemplos de itens da escala são: alguns alunos gostariam que sua aparência física fosse diferente, alguns alunos fazem muito bem sua tarefa escolar, alguns alunos se saem muito bem em qualquer tipo de esporte, alguns alunos arrumam confusão por causa das coisas que fazem alguns alunos têm uma porção de amigos.

\section{ProcedimentoseA nálisededados}

Contataram-se escolas de ensino fundamental, públicas e privadas, com vistas a explicar o objetivo do projeto e descrever as atividades a serem desenvolvidas com os alunos. Após autorização por parte das escolas para realização do estudo, o projeto foi encaminhado ao Comitê de Ética em Pesquisa de uma universidade federal. Em seguida à aprovação do projeto pelo comitê (registro no 077/2007), agendou-se com as escolas um dia para aplicação dos instrumentos. Estes foram aplicados coletivamente por alunos treinados do curso de Psicologia - bolsistas de iniciação científica e alunos matriculados em pesquisa. D elineamento fatorial e análises de variância e de correlação foram utilizados para atender aos objetivos propostos.

\section{Resultados e discussão}

Quanto à percepção dos alunos sobre o clima de sala de aula para criatividade, Autopercepção do Aluno em Relação à Criatividade foi 0 fator mais bem avaliado tanto no que diz respeito à disciplinas de Língua Portuguesa $(\mathrm{M}=3,88 ; \mathrm{DP}=0,86)$ quanto à de Matemática $(\mathrm{M}=3,77 ; \mathrm{DP}=0,95)$. 0 fator Postura do Professor em Sala de Aula na disciplina de Língua Portuguesa foi o segundo mais bem avaliado pelos alunos $(\mathrm{M}=3,64 ; \mathrm{DP}=1,02) .0$ mesmo não ocorreu quanto à avaliação desse fator considerando a disciplina de Matemática, cuja média foi a mais baixa de todos os fatores avaliados $(\mathrm{M}=3,15 ; \mathrm{DP}=1,08)$. Por outro lado, a média do fator Interesse do Aluno pela Aprendizagem em Matemática foi superior $(\mathrm{M}=3,72 ; \mathrm{DP}=0,93)$ à de Língua Portuguesa $(\mathrm{M}=3,61 ; \mathrm{DP}=0,88)$. Entretanto, quando se comparam as médias do clima de sala de aula para criatividade na disciplina de Língua Portuguesa e Matemática, não se encontram diferenças significativas na avaliação discente. A Matemática é considerada por muitos alunos como uma área difícil, impossivel de aprender, "bicho papão" ou para gênios 
(Martins, 1yyy; santos \& Diniz, 2UU4), embora represente um importante papel no processo de desenvolvimento científico e tecnológico, além de ser requisitada para a admissão nas principais instituições de ensino, bem como na maioria das ocupações profissionais. A crença de que não é possível ser criativo na Matemática também é amplamente difundida (Gontijo \& Fleith, 2009). Contudo, os resultados obtidos não apoiam essa perspectiva. Podese hipotetizar que mudanças na formação e capacitação desses professores têm promovido situações em sala de aula mais motivadoras e desafiadoras, de forma a contornar a crença da disciplina como uma área árida e pouco criativa. Em investigação prévia na qual se comparou a percepção de alunos do Distrito Federal e de Minas Gerais acerca do clima criativo em sala de aula nas disciplinas de Língua Portuguesa e Matemática (Gonçalves, Fleith \& Libório, 2011), achados semelhantes a este estudo foram obtidos quanto à amostra do Distrito Federal - nenhuma diferença significativa. Já os alunos de Minas Gerais avaliaram melhor a disciplina de Matemática.

Em relação ao autoconceito, as médias mais altas foram nas dimensões autoestima global $(\mathrm{M}=3,15$; $\mathrm{DP}=0,64)$ e conduta comportamental $(\mathrm{M}=2,94$; $\mathrm{DP}=0,63)$, ao passo que as mais baixas foram na competencia escolar $(\mathrm{IV}=2,68 ; \mathrm{DP}=0,78)$ e atlética $(\mathrm{M}=2,69 ; \mathrm{DP}=0,71)$. As dimensões aceitação social $(\mathrm{M}=2,83 ; \mathrm{DP}=0,69)$ e aparência física obtiveram respectivamente médias de 2,83 ( $\mathrm{DP}=0,69)$ e 2,82 $(\mathrm{DP}=0,81)$.

Os resultados da ANOVA indicaram diferenças significativas entre alunos de escolas públicas e particulares em relação aos fatores Postura do Professor em Sala de Aula $(F[1,466]=155,0 ; p=0,0001)$, Interesse do Aluno pela Aprendizagem $(F[1,475]=33,33 ; p=0,0001)$ e Autopercepção do Aluno com Relação à Criatividade $(\mathrm{F}[1,456]=33,88 ; \mathrm{p}=0,0001)$ da escala sobre o clima de sala de aula para criatividade na disciplina de Língua Portuguesa. Os alunos de escolas particulares avaliaram de forma mais positiva esses fatores quando comparados aos de escolas públicas. Em relação ao clima de sala de aula para criatividade na disciplina de Matemática, os resultados da análise indicaram diferenças significativas entre alunos de escolas públicas e particulares apenas no fator Interesse do Aluno pela Aprendizagem $(F[1,471]=8,76 ; \quad p=0,003)$. Os alunos de escolas públicas apresentaram uma percepção mais favorável desse fator em comparação aos de escolas particulares (veja Tabela 1).

Tabela 1. Dados dos alunos na Escala sobre o Clima Criativo por disciplina e escola

\begin{tabular}{|c|c|c|c|c|c|}
\hline \multirow{2}{*}{ Fatores } & \multirow{2}{*}{$\begin{array}{l}\text { Tipo de } \\
\text { escola }\end{array}$} & \multicolumn{2}{|c|}{ Língua Portuguesa } & \multicolumn{2}{|c|}{ Matemática } \\
\hline & & $\mathrm{M}$ & $\mathrm{DP}$ & $\mathrm{M}$ & $\mathrm{DP}$ \\
\hline \multirow[t]{2}{*}{ Postura do Professor em Sala de Aula } & Pública & 2,65 & 0,82 & 3,05 & 1,09 \\
\hline & Particular & 3,67 & 0,94 & 2,98 & 0,98 \\
\hline \multirow[t]{2}{*}{ Interesse do Aluno pela Aprendizagem } & Pública & 3,41 & 0,88 & 3,83 & 0,89 \\
\hline & Particular & 3,87 & 0,82 & 3,57 & 0,96 \\
\hline \multirow[t]{2}{*}{ Autopercepçáo do Aluno em Relaçáo à Criatividade } & Pública & 3,56 & 0,82 & 3,66 & 0,90 \\
\hline & Particular & 4,00 & 0,76 & 3,59 & 0,92 \\
\hline
\end{tabular}

No estudo realizado por Fleith e Alencar (2006), em que foi examinada a percepção de alunos do $5^{\circ}$ ano quanto ao clima de sala de aula para criatividade, observou-se que os alunos de escolas particulares tinha uma percepção mais positiva desse clima do que os de escolas públicas. Achados semelhantes foram encontrados por Castro e Fleith (2008). Nakano e Wechsler (2006), bem como Fleith e Alencar (2008), observaram desempenho superior em medidas de criatividade por parte de alunos do ensino fundamental matriculados em escolas particulares em comparação aos que frequentavam escolas públicas. 0 sistema educacional brasileiro revela uma discrepância em termos de recursos, instalações, formação continuada de professores e atividades extraclasse entre instituições públicas e particulares, o que pode influenciar nas práticas implementadas em sala de aula.

Quanto ao autoconceito, os resultados revelaram diferenças significativas entre alunos de escolas públicas e particulares em relação a todas as dimensões, exceto aparência física. Os alunos de escolas particulares apresentaram uma percepção de si mesmos mais positiva do que os de escolas públicas (veja Tabela 2). Os alunos de escolas particulares, em sua maioria, são provenientes de famílias com poder aquisitivo mais alto, o que pode significar acesso a um número maior de atividades extracurriculares, de recursos tecnológicos e de oportunidades e experiências diversificadas, contribuindo para o desenvolvimento de um autoconceito positivo em várias dimensões. 
Tabela 2. Dados dos alunos nos fatores da Escala de Autoconceito por tipo de escola

\begin{tabular}{llcccc}
\hline Fatores & Tipo de escola & M & DP & F & F \\
\hline Competência escolar & Pública & 2,55 & 0,69 & 24,02 & 0,0001 \\
& Particular & 2,86 & 0,67 & & \\
\hline Aceitaçáa social & Pública & 2,78 & 0,76 & 4,19 & 0,04 \\
& Particular & 2,89 & 0,72 & & \\
\hline Competência atlética & Pública & 2,64 & 0,69 & 3,64 & 0,06 \\
& Particular & 2,75 & 0,72 & & \\
\hline Aparéncia física & Pública & 2,73 & 0,78 & 9,165 & 0,003 \\
& Particular & 2,93 & 0,83 & & \\
\hline Conduta comportamental & Pública & 2,86 & 0,63 & 10,55 & 0,001 \\
& Particular & 3,06 & 0,60 & & \\
\hline Autoestima global & Pública & 3,06 & 0,66 & 14,962 & 0,0001 \\
& Particular & 3,27 & 0,60 & & \\
\hline
\end{tabular}

No que diz respeito à comparação entre alunos do sexo masculino e feminino, os resultados não indicaram diferenças significativas entre os dois grupos em relação a nenhum dos fatores medidos pela Escala sobre Clima para Criatividade em Sala de Aula tanto na disciplina Língua Portuguesa quanto na de Matemática. Estudos conduzidos anteriormente com alunos de $5^{\circ}$ ano do ensino fundamental também obtiveram resultados semelhantes (Matos \& Fleith, 2006; Pinheiro-Cavalcanti, 2009). Uma explicação para esse resultado decorre de mudanças na formação inicial e continuada do professor, em que o componente criatividade tem sido mais valorizado e enfatizado no contexto escolar, com reflexos nas práticas pedagógicas adotadas. Em um estudo conduzido por Godinho, Ristoff, Fontes, Xavier e Sampaio (2005) que avaliou o desempenho de alunos e alunas de $5^{\circ}$ e $9^{\circ}$ ano do ensino fundamental e $3^{0}$ ano do ensino médio, nas disciplinas de Língua Portuguesa e Matemática, com base nos dados do Sistema de Avaliação da Educação Básica (SAEB), constatou-se "um melhor desempenho das meninas em Língua Portuguesa e dos meninos em Matemática, embora as diferenças entre meninos e meninas nas áreas de Matemática sejam bem menores que em Português" (p. 42). Entretanto, o desempenho das alunas do ensino médio é um pouco acima do dos alunos nas duas disciplinas. Para os autores do estudo, "os resultados praticamente se igualam" (p. 43).

Quanto ao autoconceito, diferenças significativas foram identificadas entre alunos do gênero masculino e feminino em quatro dimensões da escala Perfil de Autopercepção para Crianças: aceitação social, competência atlética, aparência física e conduta comportamental. As alunas apresentaram uma percepção mais positiva de si mesmas apenas no que diz respeito à conduta comportamental. Nas outras dimensões, as médias dos alunos foram mais elevadas em comparação às das alunas (veja Tabela 3). Na dimensão autoestima global, observou-se uma interação significativa entre tipo de escola e gênero. Alunas de escolas públicas apresentam autoestima global mais elevada do que as que frequentam escolas particulares, ao passo que alunos de instituições particulares se percebem de forma mais positiva do que os de escolas públicas. Fernandes, Bartholomeu, Rueda, Suehiro e Sisto (2005), ao compararem o autoconceito de alunos e alunas com idade média de 9 anos, observaram que a autoimagem social e pessoal dos alunos era mais positiva do que a das alunas.

Segundo Esquivel e Hodes (2003), a sociedade, a família e o contexto de sala de aula são importantes ambientes para definição dos padrões de comportamentos socialmente aceitos para cada gênero, que via de regra impõem ao gênero feminino comportamentos mais passivos, submissos e adaptáveis às conformações sociais. Starko (1995) ressalta que pesquisas têm revelado que aos alunos é dada mais liberdade para desviarem-se das regras e encontrarem soluções mais inovadoras, ao passo que as alunas são mais criticadas e orientadas a seguirem regras e a serem menos assertivas. A longo prazo, tais experiências podem minar a confiança, iniciativa e ambição feminina. 
Tabela 3. Dados dos alunos nos fatores da Escala de Autoconceito por gênero

\begin{tabular}{llcccc}
\hline Fatores & Gênero & M & DP & F & F \\
\hline Competência escolar & Masculino & 2,74 & 0,68 & 3,70 & 0,06 \\
& Feminino & 2,63 & 0,71 & & \\
Aceitaçãáo social & Masculino & 2,90 & 0,67 & 6,76 & 0,01 \\
& Feminino & 2,77 & 0,71 & & \\
Competência atlética & Masculino & 2,84 & 0,66 & 18,87 & 0,0001 \\
& Feminino & 2,56 & 0,72 & & \\
Aparência física & Masculino & 2,96 & 0,76 & 16,11 & 0,0001 \\
& Feminino & 2,70 & 0,82 & & \\
\hline Conduta comportamental & Masculino & 2,79 & 0,60 & 26,44 & 0,0001 \\
& Feminino & 3,06 & 0,62 & & \\
\hline Autoestima global & Masculino & 3,12 & 0,61 & 0,12 & 0,73 \\
& Feminino & 3,17 & 0,68 & & \\
\hline
\end{tabular}

Os resultados apontaram ainda uma correlação positiva significativa entre percepção do clima de sala de aula para criatividade e autoconceito, especialmente nas dimensões competência escolar, conduta comportamental, autoestima global e aparência física (veja Tabela 4). Conforme defendido por vários autores (Chávez-Eakle, Lara \& Cruz-Fuentes, 2006; Esquivel \& Hodes, 2003; Nickerson, 1999; Runco, 2007), existe uma associação entre autoimagem e criatividade. 0 indivíduo que acredita nos seus recursos internos tem muito mais chance de criar ou transformar a realidade
(Wechsler, 1993, p. 76). Segundo Cropley (2005), métodos de ensino que enfatizam criatividade podem ter efeitos benéficos sobre atitudes em relação a escola, motivação e autoconceito dos alunos. Meador, Fishkin e Hoover (1999) relatam evidências empíricas de aumento no autoconceito de alunos que foram expostos a estratégias e programas de criatividade. Contudo, é importante destacar que os índices de correlação obtidos no presente estudo são muito baixos, indicando uma correlação significativa, porém fraca.

Tabela 4. Índices de correlação entre fatores de Autoconceito e Clima para Criatividade em Sala de Aula

\begin{tabular}{lcccccc}
\hline & $\begin{array}{c}\text { Competência } \\
\text { escolar }\end{array}$ & $\begin{array}{c}\text { Aceitação } \\
\text { social }\end{array}$ & $\begin{array}{c}\text { Competência } \\
\text { atlética }\end{array}$ & $\begin{array}{c}\text { Aparência } \\
\text { física }\end{array}$ & $\begin{array}{c}\text { Conduta } \\
\text { comport. }\end{array}$ & $\begin{array}{c}\text { Autoestima } \\
\text { global }\end{array}$ \\
\hline Postura - professor LP & $0,19^{* *}$ & $0,14^{* *}$ & $0,10^{*}$ & $0,13^{* *}$ & $0,21^{* *}$ & $0,14^{* *}$ \\
Interesse - aluno LP & $0,28^{* *}$ & 0,09 & 0,05 & $0,13^{* *}$ & 0,09 & $0,13^{* *}$ \\
Autopercepção - aluno LP & $0,31^{* *}$ & $0,18^{* *}$ & $0,17^{* *}$ & $0,19^{* *}$ & $0,26^{* *}$ & $0,19^{* *}$ \\
Postura - professor M & $0,21^{* *}$ & 0,03 & $-0,42$ & 0,69 & 0,07 & 0,08 \\
Interesse - aluno M & $0,29^{* *}$ & $-0,02$ & $-0,02$ & $0,10^{*}$ & $0,20^{* *}$ & $0,12^{*}$ \\
Autopercepção - aluno M & $0,30^{* *}$ & 0,09 & 0,09 & $0,21^{* *}$ & $0,16^{* *}$ & $0,18^{* *}$ \\
\hline
\end{tabular}

Nca: LP= Língua Portuguesa; $\mathrm{M}=$ Matemática.

$* p<0,05 ; * * p<0,01$

\section{Considerações finais}

Os resultados revelam que os alunos do $6^{\circ}$ ano do ensino fundamental, participantes do estudo, têm uma percepção favorável do clima de sala de aula para criatividade nas disciplinas de Língua Portuguesa e Matemática, bem como um autoconceito positivo. Grandes diferenças de avaliação foram encontradas entre alunos de escolas públicas e particulares, apresentando os últimos, de maneira geral, médias mais elevadas. Os dados sinalizam ainda um autoconceito mais positivo pelos alunos do gênero masculino quando comparados aos do gênero feminino especialmente no que diz respeito ao corpo (por exemplo, aparência física e competência atlética) e habilidades sociais. Não foram observadas diferenças de gênero ou de disciplina quanto à percepção do clima de sala de aula. Correlações positivas significativas, porém fracas, foram identificadas entre autoconceito e clima para criatividade em sala de aula. É possível que essa relação não seja linear, mas mediada por outras variáveis como, por exemplo, a motivação do aluno. Hennessey e Amabile (1988) afirmam que a variável autoconceito desempenha um papel importante nos processos motivacionais e criativos. Segundo Csikszentmihalyi (1996), quando o indivíduo se encontra em um estado de total envolvimento no processo criativo, ele não se preocupa com erros ou insucessos. Vale lembrar que a amostra de conveniência utilizada no estudo pode ser um fator limitador do poder de generalização dos resultados. 
Por outro lado, os resultados obtidos podem contribuir na elaboração de cursos de formação inicial e continuada de professores da educação básica, de planos de aulas, avaliação do desempenho discente, bem como nortear o desenvolvimento de práticas pedagógicas que despertem no aluno o prazer pela aprendizagem e pelo ato de criar, fortalecendo uma autoimagem positiva. Como sugestões para futuras pesquisas, indica-se a realização de estudos que envolvam observação e avaliação das atividades implementadas em sala de aula quanto ao nível de estimulação da criatividade e envolvimento do aluno, estudos de casos de alunos e professores muito criativos, comparação do clima de sala de aula entre disciplinas distintas, análise de fatores de instituições escolares e sua relação com a criatividade docente e discente, e exame de efeitos de treinamento de criatividade em variáveis como autoconceito e motivação para aprendizagem.

\section{Referências}

Alencar, E. M. L. S., \& Fleith, D. S. (2004). Creativity in university courses: perceptions of professors and students. Gifted and Talented Intemational, 19(1), 24-28.

Alencar, E. M. L. S., \& Fleith, D. S. (2009). Criatividade

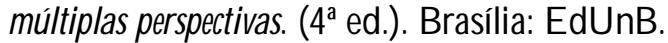

Amabile, T. A. (1996). Creativityin context. Boulder, CO : Westview Press.

Bandeira, D. R., Arteche, A. X., \& Reppold, C. T. (2008). Escala de autopercepção de Harter para adolescentes: um estudo de validação. Psicdoga: Teria ePesquisa, 24(3), 341-345.

Beghetto, R. A. (2009). In search of the unexpected: finding creativity in the micromoments of the classroom. Psychdogy of Aesthetics, Creativity, and the Arts, 3(1), 2-5.

Byrne, B. M. (1996). Measuing sedf-coneqt across life span Washington, DC: American Psychological Association.

Castro, J. S. R., \& Fleith, D. S. (2008). Criatividade escolar: relação entre tempo de experiência docente e tipo de escola. Psicloja Escdar e Eduradional, 12(1), 101-118.

Chávez-Eakle, R. A., Lara, M. C., \& Cruz-Fuentes, C. (2006). Personality: a possible bridge between creativity and psychopathology? Creativity Reserch Jaumal, 18(1), 27-38.
Cropley, A. J. (2005). Creativityin eeturationandleaming A guide for teachess and elucators Londres: RoutledgeFalmer.

Csikszentmihalyi, M. (1996). Creativity. Nova Iorque: HarperCollins.

Csikszentmihalyi, M. (1999). Implications of a systems perspective for the study of creativity. Em R. J. Sternberg (O rg.), Handbook of crativity (pp. 313335). Nova Iorque: Cambridge University Press.

Esquivel, G. B., \& Hodes, T. G. (2003). Creativity, development, and personality. Em J. Houtz (O rg.), The edurational psychdogy of ceativity (pp. 135-165). Cresskill, NJ: Hampton.

Fernandes, D. C., Bartholomeu, D., Rueda, F. J., Suehiro, A. C. B., \& Sisto, F. F. (2005). Autoconcepto y rasgos de personalidad: un studio correlacional. Psicdoja Escolar e Educadional, 9(1), 15-25.

Fleith, D. S., \& Alencar, E. M. L. S. (2005). Escala sobre o clima para criatividade em sala de aula. Psicloga: Teria ePesquisa, 21(1), 85-91.

Fleith, D. S., \& Alencar, E. M. L. S. (2006). Percepção de alunos do ensino fundamental quanto ao clima de sala de aula para criatividade. Psicdoja $\mathbf{m}$ Estudo 11(3), 513 - 521.

Fleith, D. S., \& Alencar, E. M. L. S. (2008). Características personológicas e fatores ambientais relacionados à criatividade do aluno do ensino fundamental. AvaliaçãoPsicdóġa, 7(1), 35-44.

Fleith, D. S., Almeida, L. S., \& Peixoto, F. J. B. (2011). Estudo de validação da Escala Clima para Criatividade em Sala de Aula. Estudos de Psicdogia, 28(3), 307-314.

Fleith, D. S., Renzulli, J. S., \& Westberg, K. L. (2002). Effects of a creativity training program on divergent thinking abilitied and self-concept in monolingual and bilingual classrooms. Creativity ReserchJaumal, 14(3 \& 4), 373-386.

Fraser, B. J. (1984). Differences between preferred and actual classroom environment as perceived by primary students and teachers. British Jamal of Edurational Psychdogy, 54(3), 336-339.

Gall, M. D., Borg, W. R., \& Gall, J. P. (1996). Educational rearch (6 $6^{\mathrm{a}}$ ed.). White Plains, NY: Longman.

Godinho, T., Ristoff, D., Fontes, A., Xavier, I. M., \& Sampaio, C. E. M. (2005). Trajeónia da milher na exuraçãobrasilèra: 1996-2003. Brasília: INEP. 
Gonçalves, F. C., Fleith, D. S., \& Libório, A. C. O. (2011). Criatividade em aula: percepção de alunos de dois estados brasileiros. Arquivos Brasileros de Psicdoga, 63(1), 22-30.

Gontijo, C. H., \& Fleith, D. S. (2009). Motivação e criatividade em matemática: um estudo comparativo entre alunas e alunos de ensino médio. Educacãa Temática Digital, 10(número especial), 147-167.

Harter, S. (1985). Manual for the sedf-pereption profile for dildren Manuscrito não publicado. University of D enver, D enver, CO.

Harter, S. (1990). Issues in the assessment of selfconcept of children and adolescents. Em A. LaG reca (O rg.), Through the eyes of a dild (pp. 292325). Boston: Allyn \& Bacon.

Hennessay, B. A., \& Amabile, T. M. (1988). The conditions of creativity. Em R. J. Sternberg (O rg.), The mature of ceativity (pp. 11-38). Nova Iorque: Cambridge University Press.

Johnson, W. L., \& Johnson, A. M. (1999). Assessing perception of school climate among Jordanian students in English-speaking schools. Psydhdojcal Reports, 84(2), 395-397.

Martins, U. P. (1999). Matemática: quelido papão é sse? (Dissertação de Mestrado). Universidade Federal de Mato Grosso, Cuiabá, Mato Grosso, Brasil.

Matos, D. R., \& Fleith, D. S. (2006). Criatividade e clima criativo entre alunos de escolas abertas, intermediárias e tradicionais. Psiødoga Escdar e Eduradional, 10(1), 109-120.

Meador, K. S., Fishkin, A. S., \& Hoover, M. (1999). Research-based strategies and programs to facilitate creativity. Em A. S. Fishkin, B. Cramond \& P. Olszewski-Kubilius (O rgs.), Investigating arativity in youth: rearch and mothods (pp. 389-415). Cresskill, NJ: Hampton Press.

Nakano, T. C., \& Wechsler, S. M. (2006). Teste Brasileiro de Criatividade Figural: proposta de normas. AvaliaçãoPsicó́gica, 5(2), 159-170.

Nickerson, R. S. (1999). Enhancing creativity. Em R. J. Sternberg (O rg.), Handbook of cativity (pp. 392350). Nova Iorque: Cambridge Academic Press.
O urofino, V. T. T., \& Fleith, D. S. (2005). Um estudo comparativo sobre a dupla excepcionalidade: superdotação/ hiperatividade. Avaliação Psiđóǵca, 4(2), 165-182.

Pinheiro-Cavalcanti, M. M. (2009). Rdaç̃eses entre motivação para aprendar, percepão do dima de sala de aula para ciatividadee desempenho esclar dealunos db50 anodoensino fundamental (D issertação de Mestrado). Universidade de Brasília, Brasília-DF, Brasil.

Runco, M. A. (2007). Creativity. San Diego, CA: Elsevier.

Santos, N. A. P., \& Diniz, M. I. S. V. (2004). As concepcóes dos alunos ao final da escla básica podkm explicar parque des não querem aprender. Trabalho apresentado no VIII Encontro Nacional de Educação Matemática, Recife, Pernambuco.

Selby, E. C., Shaw, E. J., \& Houtz, J. C. (2005). The creative personality. Gifted Child Quartely, 49(4), 300-314.

Silva, S. S. (2002). Desemperho esclar e autoconcito de cianças atendidas emumserviço psicopetagógica pereppãa de alunos, mães, professoras, psicóogas e pechagogas (Dissertação de Mestrado). Universidade de Brasília, Brasília-DF, Brasil.

Starko, A. J. (1995). Creativity in the dassrom White Plains, NY: Longman.

Valentini, N. C., Villwock, G., Vieira, L. N., Vieira, J. L. L., \& Barbosa, M. L. L. (2010). Validação brasileira da Escala de Autopercepção de Harter para crianças. Psiđogja: RellexãoeCńtica, 23(3), 411-419.

Wechsler, S. M. (1993). Criatividade Descdanindb e encorajanda Campinas, SP: Psy.

Wechsler, S. M. (2006). Validity of the Torrance Tests of Creative Thinking to the brazilian culture. CreativityRearchJaumal, 18(1), 15-25.

Wechsler, S. M., \& Souza, V. L. T. (Org.). (2011). Criatividade e aprendizagm Caminhos e descobatas em pespetivaintemacional. São Paulo: Loyola.

Reedidbem30/ 11/ 2011

Reformuladbem05/ 05/ 2012

Aprovadoem06/ 06/ 2012 
Apoio: Conselho Nacional de Desenvolvimento Científico e Tecnológico (CNPq).

Sobre as autoras:

Denise de Souza Fleith é psicóloga e Ph.D. em Psicologia Educacional pela University of Connecticut. Realizou seu pós-doutorado no National Academy for Gifted and Talented Youth (University of Warwick) em 2005. É professora associada do Departamento de Psicologia Escolar e do D esenvolvimento do Instituto de Psicologia da Universidade de Brasília. É orientadora de mestrado e doutorado no Programa de Pós-Graduação em Processos de Desenvolvimento Humano e Saúde. É pesquisadora do CNPq e editora associada da revista Psicologia: Teoria e Pesquisa.

E unice M. L. Soniano de Alencar é psicóloga e Ph.D. em Psicologia pela University of Purdue. Foi post-doctoral scholar no Gifted Education Resource Institute, nos Estados Unidos. É professora emérita da Universidade de Brasília, pesquisadora do $\mathrm{CNPq}$, cidadã honorária de Brasília, membro da Academia Paulista de Psicologia e membro honorário do Conselho Brasileiro para Superdotação. Participa do conselho editorial de periódicos científicos de distintos países.

Contato com as autoras:

Universidade de Brasília, Instituto de Psicologia - Campus Darcy Ribeiro. CEP: 70910-900. Brasília-DF.

E-mail: fleith@unb.br 